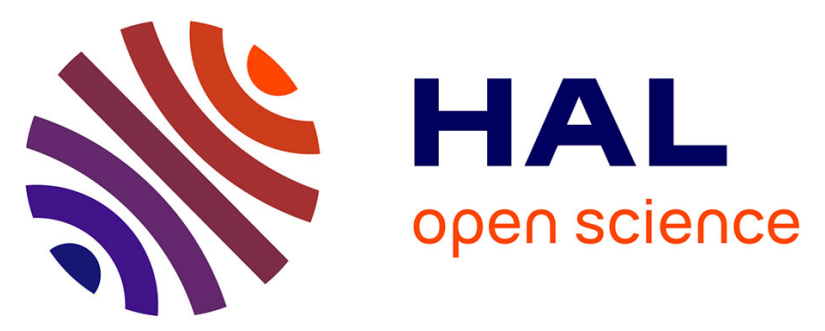

\title{
Geometry and loading effects on performances of mode-stirred reverberation chambers: an experimental study
}

Ludivine Le Bars, Jean-François Rosnarho, Philippe Besnier, Jérôme Sol, Francois Sarrazin, Elodie Richalot

\section{To cite this version:}

Ludivine Le Bars, Jean-François Rosnarho, Philippe Besnier, Jérôme Sol, Francois Sarrazin, et al.. Geometry and loading effects on performances of mode-stirred reverberation chambers: an experimental study. 2018 International Symposium on Electromagnetic Compatibility - EMC EUROPE, Sep 2019, Barcelona, Spain. 10.1109/EMCEurope.2019.8872134 . hal-02282528

\section{HAL Id: hal-02282528 \\ https://hal.science/hal-02282528}

Submitted on 10 Sep 2019

HAL is a multi-disciplinary open access archive for the deposit and dissemination of scientific research documents, whether they are published or not. The documents may come from teaching and research institutions in France or abroad, or from public or private research centers.
L'archive ouverte pluridisciplinaire HAL, est destinée au dépôt et à la diffusion de documents scientifiques de niveau recherche, publiés ou non, émanant des établissements d'enseignement et de recherche français ou étrangers, des laboratoires publics ou privés. 


\section{Geometry and loading effects on performances of mode-stirred reverberation chambers: an experimental study}

\author{
Ludivine Le Bars, Jean-François \\ Rosnarho \\ SIEPEL : Société Industrielle d'Etudes et \\ Protections Electroniques, Impasse de la \\ Manille, F-56470 \\ La Trinité-sur-Mer, France
}

\author{
Philippe Besnier, Jérôme Sol \\ INSA Rennes, CNRS, IETR - UMR \\ 6164, F-35000 \\ Rennes, France
}

\author{
François Sarrazin, Elodie Richalot \\ Université Paris-Est, ESYCOM (FRE2028), \\ CNAM, CNRS, ESIEE-Paris, Université Paris- \\ Est Marne-la-Vallée, F-77454 \\ Marne-la-Vallée, France
}

\begin{abstract}
In this paper, we aim at highlighting the specific roles of the geometry and loading in a mode-stirred reverberation chamber. A geometrical modification is achieved through the introduction of several metallic caps on the cavity walls, in order to fulfill chaoticity requirements. Loading modifications are achieved by putting absorbers on ground. Tests have been performed in three reverberation chambers of different dimensions, and thus, of different lowest usable frequencies. Respective and combined roles of both types of modification are analyzed and clarified.
\end{abstract}

\section{Keywords-chaoticity, absorption, reverberation chamber}

\section{INTRODUCTION}

Reverberation chambers ( $\mathrm{RCs}$ ) are now widely used as a measurement tool to perform either electromagnetic compatibility tests or antenna measurements [1] [2].

Standards define several parameters to study a reverberation chamber in order to check the proper functioning of the system. It is commonly assumed that the standard deviation of the maximum E-field within the working volume of RC and over a complete stirrer rotation must not exceed the standard limit while respecting a minimum number of uncorrelated stirrer positions in order to obtain compliant measurements. It is however difficult to reach this maximum E-field uniformity in accordance with the standards at low frequencies for a small RC. As this study aims at decreasing the lowest usable frequency (LUF) of RCs, our frequency range of interest is around and below the announced LUF.

Though the main goal of either EN 61000-4-21 [3] or RTCA DO-160G [4] standards is to check such defined maximum E-field uniformity, it gives a poor indication on the statistics (i.e. no indication about its first moments) of the Efield itself. To refine RC analysis additional criteria are necessary. In this paper we use:

1) the standard deviation to mean ratio of the E-field over a complete stirrer rotation and considering all the probe or antenna positions, and

2) the distribution of Pnini-Shapiro for chaotic cavity

We aim at understanding the impact of the aforesaid criteria by adding several caps in the enclosure to make an RC chaotic, as well as the effect of loading it with absorbing materials.

Among many experiments performed in different RCs (ESYCOM, IETR, SIEPEL) we focus here on a limited set of significant results. First, experiments were carried out in the RC of ESYCOM laboratory comparing two configurations, an empty RC and an RC made chaotic thanks to the introduction of three metallic caps. Thus, this first measurement campaign aims at understanding the impact of the RC geometry. Second, a measurement campaign was achieved in another $\mathrm{RC}$ at SIEPEL company. It aimed at investigating the loading variation effect through adjustment to absorber types and quantities. A third campaign, not presented here, aimed at confirming the previous results in the RC of IETR laboratory. Then, we compare the chaotic and the loaded RCs at ESYCOM laboratory, in order to understand the impact of solely the RC geometry when keeping constant the quality factor of both cavities.

This communication is organized as follows. Section II describes the test environments. Section III deals with the effects of the geometry / chaoticity of the RC. Section IV is devoted to the demonstration of the impact of the load when placing an absorber in the RC. Section V compares an RC with a load and a chaotic one with similar quality factors. We then conclude in Section VI.

\section{DESCRIPTION OF TEST ENVIRONMENTS}

Three RCs were studied for this article. The first one is the enclosure of the ESYCOM laboratory (Fig. 1). Its dimensions are $2.95 \times 2.75 \times 2.35 \mathrm{~m}^{3}$, and the three caps placed on three orthogonal walls have a diameter of $1.586 \mathrm{~m}$ and high $0.28 \mathrm{~m}$. The LUF of the empty cavity (without caps), defined according to the standards, is estimated to be about $400 \mathrm{MHz}$.

SIEPEL RC dimensions are $2.48 \times 2.48 \times 2.86 \mathrm{~m}^{3}$. The three caps used for the chaotic configuration have a diameter of $1.80 \mathrm{~m}$ and high $30 \mathrm{~cm}$. The estimated LUF is about 400 $\mathrm{MHz}$ as for the ESYCOM reverberation chamber.

The third RC studied is in the IETR laboratory. Its dimensions are $8.7 \times 2.9 \times 3.7 \mathrm{~m}^{3}$. The three hemispheres placed in the RC to make it chaotic have a diameter of $50 \mathrm{~cm}$. The LUF is of $200 \mathrm{MHz}$. 


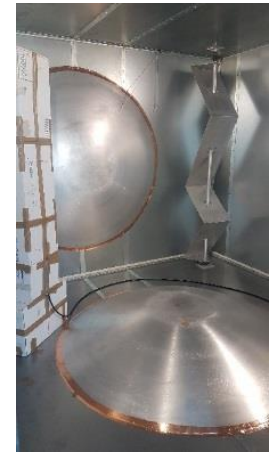

Fig.1. ESYCOM RC - Chaotic configuration $\left(2.95 \times 2.75 \times 2.35 \mathrm{~m}^{3}\right)-$ $\mathrm{LUF}=400 \mathrm{MHz}$

\section{EFFECT OF GEOMETRY}

In this section we examine the contribution of the RC chaoticity by introducing 3 caps on the cavity walls at ESYCOM laboratory.

Measurements have been performed for 60 stirrer positions and $1 \mathrm{MHz}$ frequency step by using a fixed transmitting monopole antenna and a moving receiving one to collect the data at eight positions in the RC in the $300-$ $600 \mathrm{MHz}$ frequency range. We compare the results between the empty $\mathrm{RC}$ and the chaotic one.

\section{A. E-field Statistics}

To understand the impact of the geometry modification of the $\mathrm{RC}$, we first estimate the loading effect of the inserted caps by calculating the quality factor $Q$ [7]:

$$
Q=<|S 11-<S 11>|^{2}>\frac{Z_{0} \omega \varepsilon V}{\left(\frac{\lambda^{2}}{4 \pi}\right)\left(1-|<S 11>|^{2}\right)^{2} \eta^{2}}
$$

With $S 11$ the reflection coefficient of the transmitting antenna, $Z_{0}$ the free space impedance, $V$ the cavity volume, $\eta$ the antenna efficiency.

From $Q$ we can deduce the modal overlap $d[8]$ :

$$
d=\frac{8 \pi V}{Q}\left(\frac{f}{c}\right)^{3}
$$

Finally, we estimate the standard deviation to mean ratio $\frac{\sigma}{\mu}$, namely the coefficient of variation $(C V)$ :

$$
\widehat{C V}=\frac{\sqrt{\frac{1}{n-1} \sum\left(E_{\alpha i}-<E_{\alpha}>_{n}\right)^{2}}}{<E_{\alpha}>_{n}}
$$

where $\left\langle E_{\alpha}\right\rangle_{n}$ corresponds to the ensemble average of the E-field component on a complete stirrer rotation and over all the antenna positions. For an ideal Rayleigh distribution of the E-field, the value of this ratio converges to 0.52 .

Fig. 2 shows the frequency variation of $Q$ (Fig. 2a), $d$ (Fig. 2b) and $C V$ (Fig. 2c) in the classical RC and the chaotic one. Due to strong frequency variations, all these results are averaged over a $10 \mathrm{MHz}$ sliding window.

According to this figure, the quality factor is lower in the chaotic configuration than in the empty one which implies a higher modal overlap. Indeed, the quality factor related to Joule effect on the cavity walls is proportional to $V / S$ where $V$ is the cavity volume and $S$ is the wall surfaces and the insertion of spherical caps decreases the cavity volume and increases its walls surface.
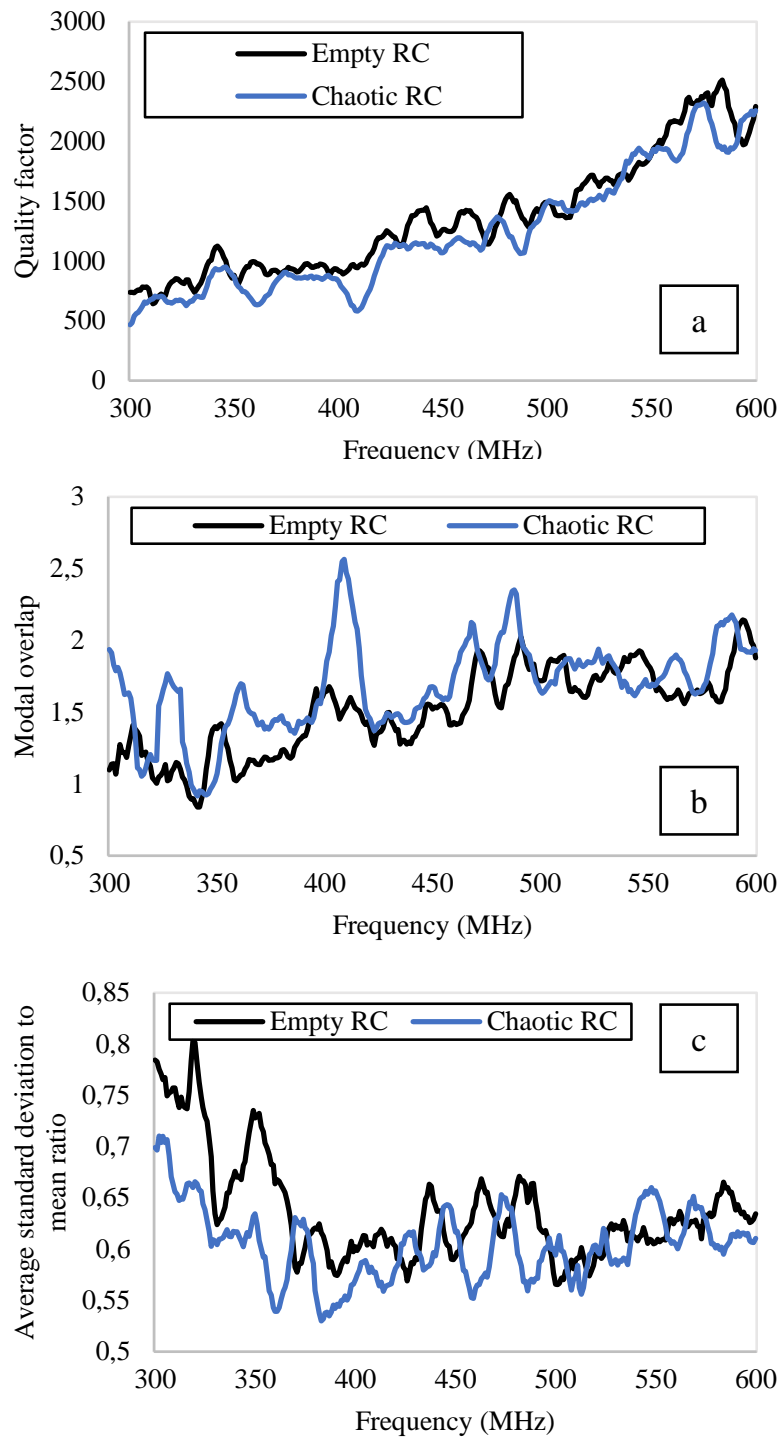

Fig.2. ESYCOM RC. (a) Quality factor. (b) Modal overlap. (c) Coefficient of variation. Results averaged over a $10 \mathrm{MHz}$ sliding window.

Considering the $C V$, the results for the chaotic configuration is lower than in the empty RC, especially in the lower frequency range. This is an indication that the E-field distribution is closer to a Rayleigh distribution when caps are present.

\section{B. Study of Normative Parameters}

In this part, we are interested in the impact of chaoticity according to two normative parameters, the number of uncorrelated stirrer positions [9] and the standard deviation of the maximum E-field as defined by the EN 61000-4-21 as follows:

$$
\sigma_{\alpha} d B=\sqrt{\frac{\sum\left(\operatorname{Emax}_{i, \alpha}-<\operatorname{Emax}_{\alpha}>_{N_{\text {probe }}}\right)^{2}}{N_{\text {probe }^{-1}}}}
$$

with Emax the maximum field amplitude over a stirrer rotation, $\alpha=x, y, z$ and $i=$ position of the antenna.

Fig. 3 shows the frequency variation of the standard deviation of maximum E-field vertical component and the number of uncorrelated stirrer positions. Though these curves are affected by statistical fluctuations, it appears that the standard deviation of the maximum E-field exhibits, on 

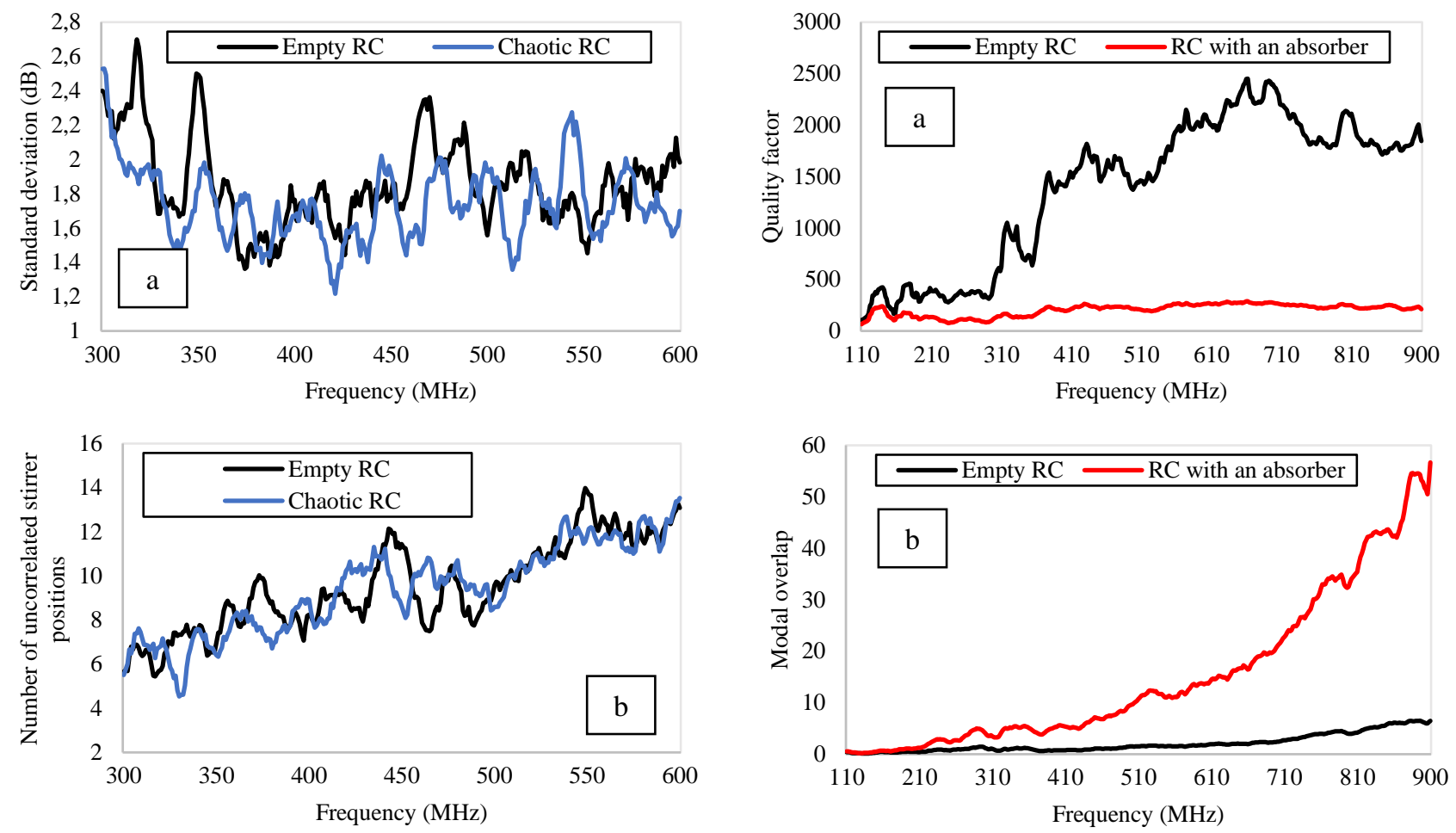

Fig.3. ESYCOM RC. (a) Standard deviation. (b) Number of uncorrelated stirrer positions. Results averaged over a $10 \mathrm{MHz}$ sliding window.

average, a better performance in the chaotic configuration in the considered 300 to $600 \mathrm{MHz}$ frequency range. Results for the number of uncorrelated stirrer positions seems to be stable.

The introduction of caps inside the RC (to make its geometry chaotic) has a positive impact on performances criteria ( $C V$ and $\sigma_{\alpha} d B$ ) but also implies a noticeable decrease of the quality factor. To understand if the observed effects are due to the geometry modifications or to the quality factor decrease, we compare in the next section an empty RC with an RC loaded by an absorber.

\section{LOADING / ABSORPTION EFFECT}

This section concerns the effect of loading / absorption in an $\mathrm{RC}$ on the same criteria as above, i.e. $Q, d, C V$ and normative criteria.

The following results correspond to the tests carried out at SIEPEL between 100 and $900 \mathrm{MHz}$ with a frequency step of $2 \mathrm{MHz}$ and for 60 stirrer positions. Data were collected at eight isotropic field probe positions without any receiving antenna in the chamber but with and without the presence of an absorber. The indicators shown have been obtained from the $x$ component of the electric field but similar results are obtained on $y$ and $z$ axes.

Fig. 4a highlights the huge decrease of the quality factor which is correlated to the large increase of the modal overlap (Fig. 4b). This has also a consequent impact on $C V$ as shown in Fig. 4c. Indeed, above $600 \mathrm{MHz}$, we reach the ideal limit of Hill's hypothesis that is a ratio of 0.52 .

However, the presence of absorbing material causes a large decrease of uncorrelated stirrer positions (Fig. 5b). Significant changes in the chamber states occur only for more distinct positions of the stirrer due to the low quality factor. Besides, the maximum E-field is more uniform. On the one

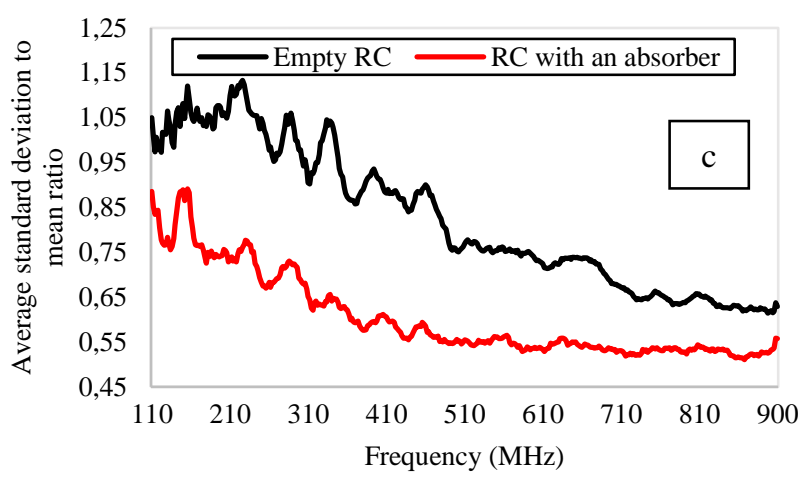

Fig.4. SIEPEL RC. (a) Quality factor. (b) Modal overlap. (c) Coefficient of variation. Results averaged over a $10 \mathrm{MHz}$ sliding window.

hand, absorbing material participates to increase the average number of modes excited at each frequency and stirrer position. On the other hand, it reduces the number of states of the chamber to an apparently still acceptable limit.

According to standards [3] [4], the RC calibration requires the use of an isotropic field probe to measure the $\mathrm{E}$ field components at the eight corners of the usable volume. In Fig. 6, we compare the standard deviation of both RC configurations at SIEPEL according to standard RTCA DO$160 \mathrm{G}$ for a frequency step of $2 \mathrm{MHz}$ without frequential averaging. We observe a significant improvement in the statistical uniformity of the field. Only 5 exceedances of the standard limit are observed when the absorbing material is present whereas 43 of them are observed for the empty chamber configuration.

Obviously, we notice that the loading factor related to the presence of these absorbing materials induces an important decrease of the power density generated in the chamber. It seems reasonable to search for a trade-off between performance improvement and quality factor preservation.

To conclude this part, we can confirm the improvement of the $C V$ while loading an RC. However, if one wishes to 

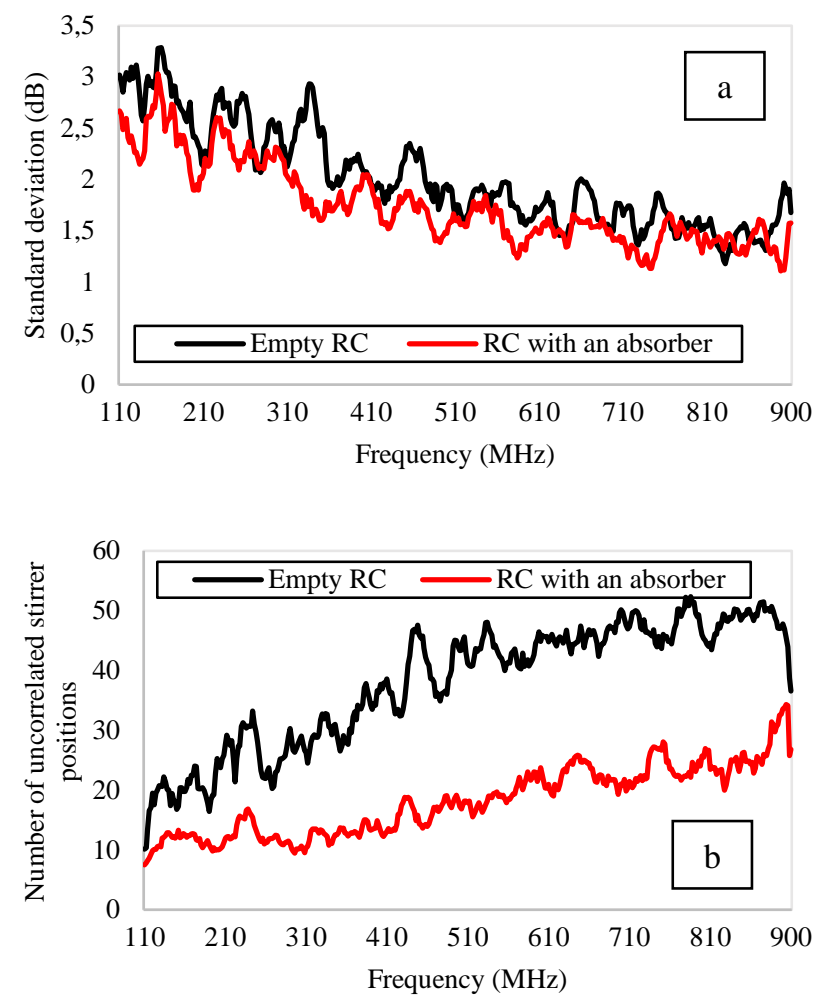

Fig.5. Measurement results at SIEPEL. (a) Standard deviation. (b) Number of uncorrelated stirrer positions. Results averaged over a $10 \mathrm{MHz}$ sliding window.

significantly reduce the field standard deviation, it is necessary in return to strongly increase the load and thus reduce the number of independent realizations. It is therefore a question of making a compromise on the load to be added as the decrease of the quality factor (i.e. increasing the
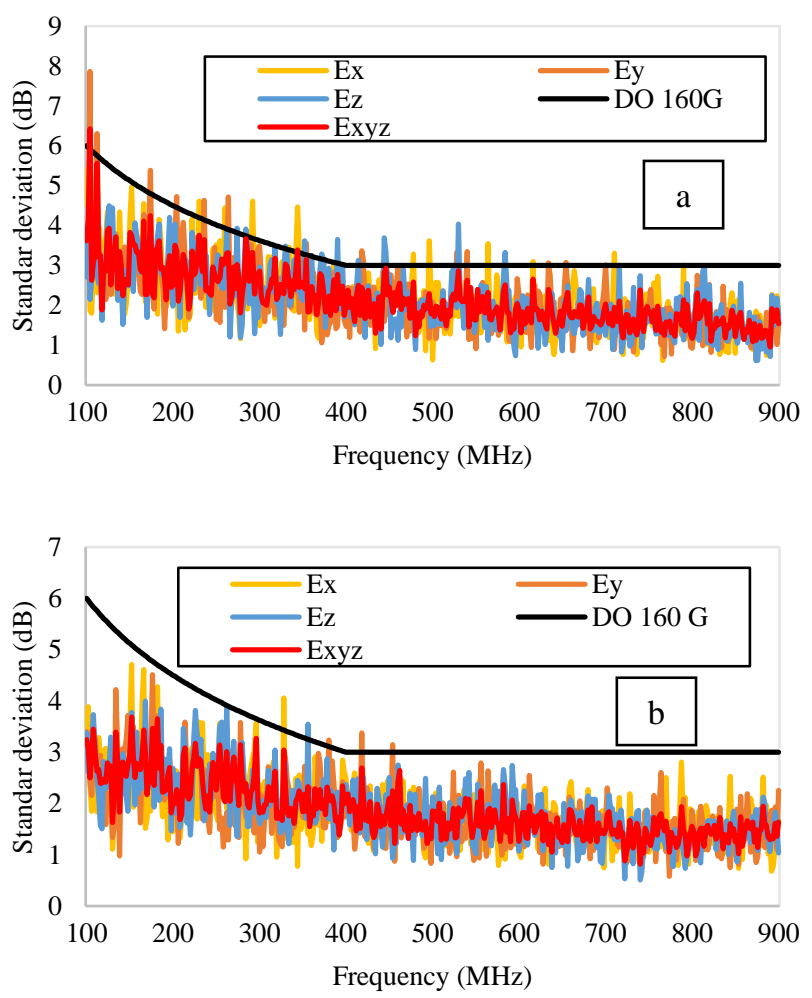

Fig.6. Standard deviation according to the RTCA DO-160G at SIEPEL. (a) Empty RC. (b) RC with an absorber. modal overlap) helps to obtain a good field uniformity and to improve the $C V$ but decreases the number of uncorrelated configurations.

\section{CHAOTICITY IMPACT}

In this section we compare the performances of the chaotic RC at ESYCOM laboratory with the one loaded by an absorber so that both cavities have the same quality factor and the only effect of the chaoticity can be put in relief. The experimental setup is the same as in the section III.

\section{A. E-field Statistics}

Fig. 7 shows the frequency variation of the quality factor, the modal overlap and the coefficient of variation $(\mathrm{CV})$ in the $\mathrm{RC}$ with an absorber and in the chaotic one. All these quantities are averaged over a $10 \mathrm{MHz}$ sliding window.

According to this figure, the quality factor is almost the same in both configurations which implies an identical modal overlap. Indeed, the modal overlap is proportional to V / Q (2), but the volume difference of classical and chaotic RCs is small $\left(19 \mathrm{~m}^{3}\right.$ for the classical RC and $18 \mathrm{~m}^{3}$ for the chaotic one) compared to the overall quality factor. Considering the $C V$, the two results are close to each other
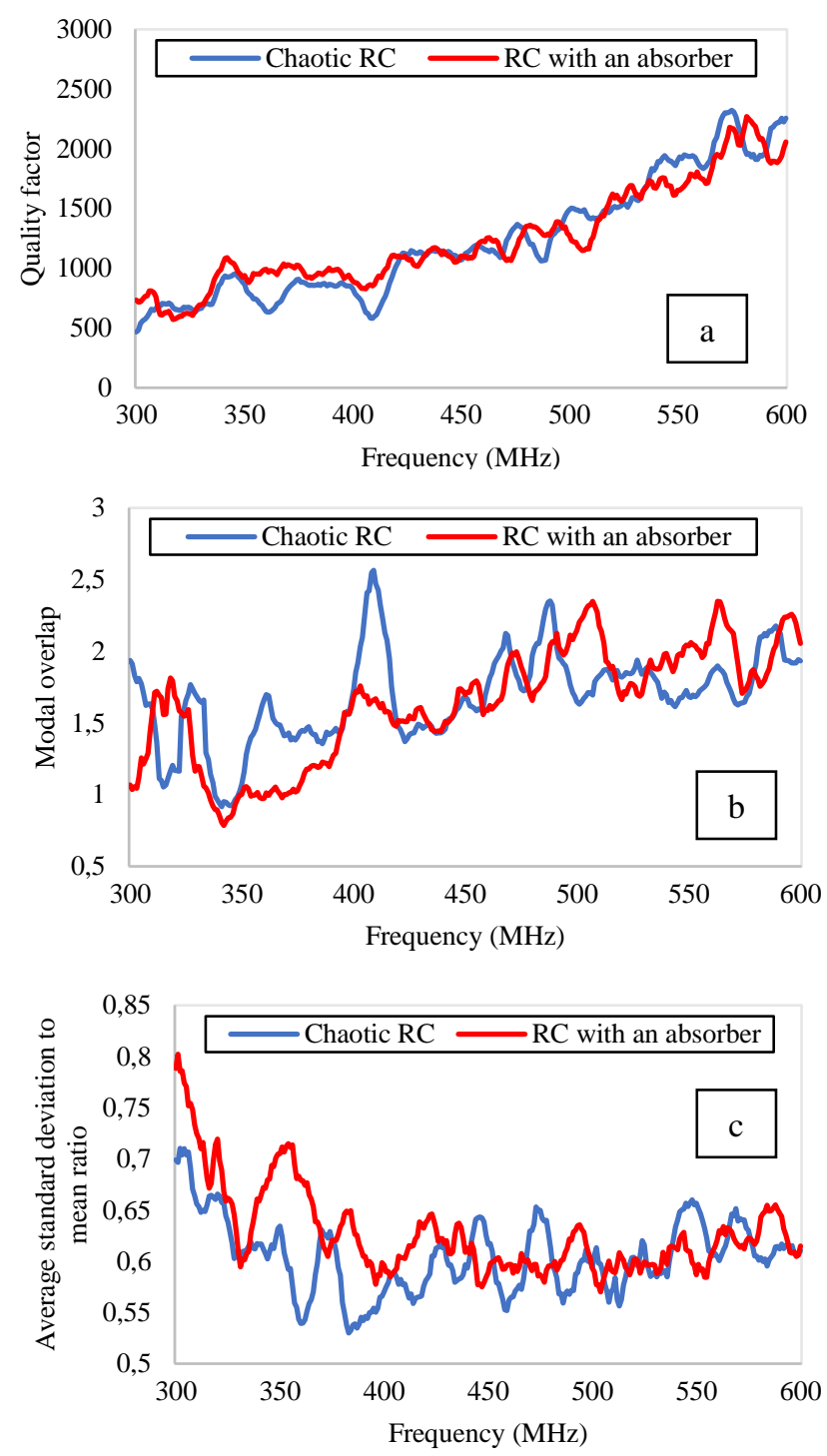

Fig.7. ESYCOM RC. (a) Quality factor. (b) Modal overlap. (c) Coefficient of variation. Results averaged over a $10 \mathrm{MHz}$ sliding window. 
from $450 \mathrm{MHz}$ and beyond. However, at low frequencies, we observe a significant decrease of this parameter in the chaotic configuration.

In the next section, we study two normative parameters, the number of uncorrelated stirrer positions and the standard deviation of maximum E-field uniformity as defined by the standards.

\section{B. Study of Normative Parameters}

Fig. 8 shows the curves of the standard deviation of maximum E-field uniformity and the number of uncorrelated stirrer positions. The addition of caps neither decreases the number of uncorrelated stirrer positions nor modifies the standard deviation criterion.

The impact of chaoticity is not significant on these normative parameters. However, it seems that below 430 $\mathrm{MHz}$ the chaotic cavity presents a slightly better maximum E-field uniformity on average. Indeed, the mean of the standard deviation between 300 and $400 \mathrm{MHz}$ is about 1.82 in the $\mathrm{RC}$ with an absorber whereas it is about 1.76 in the chaotic reverberation chamber. Nevertheless, the standard criterion is not sensitive enough to this geometrical modification. As highlighted in Fig. 7, investigation of $\mathrm{CV}$ gives a better indication of the benefit of adding chaoticity in this $300-400 \mathrm{MHz}$ frequency range.

\section{Intensity Distribution According to Pnini and Shapiro}

Let us consider the intensity distributions established by Pnini and Shapiro [5] [6] in order to study the contribution of geometry by the introduction of caps in an RC. The Pnini and Shapiro distribution depicts universal fluctuations of intensity in a chaotic cavity as a function of the so-called phase rigidity, itself related to the modal overlap (i.e. according to losses within the chaotic cavity).
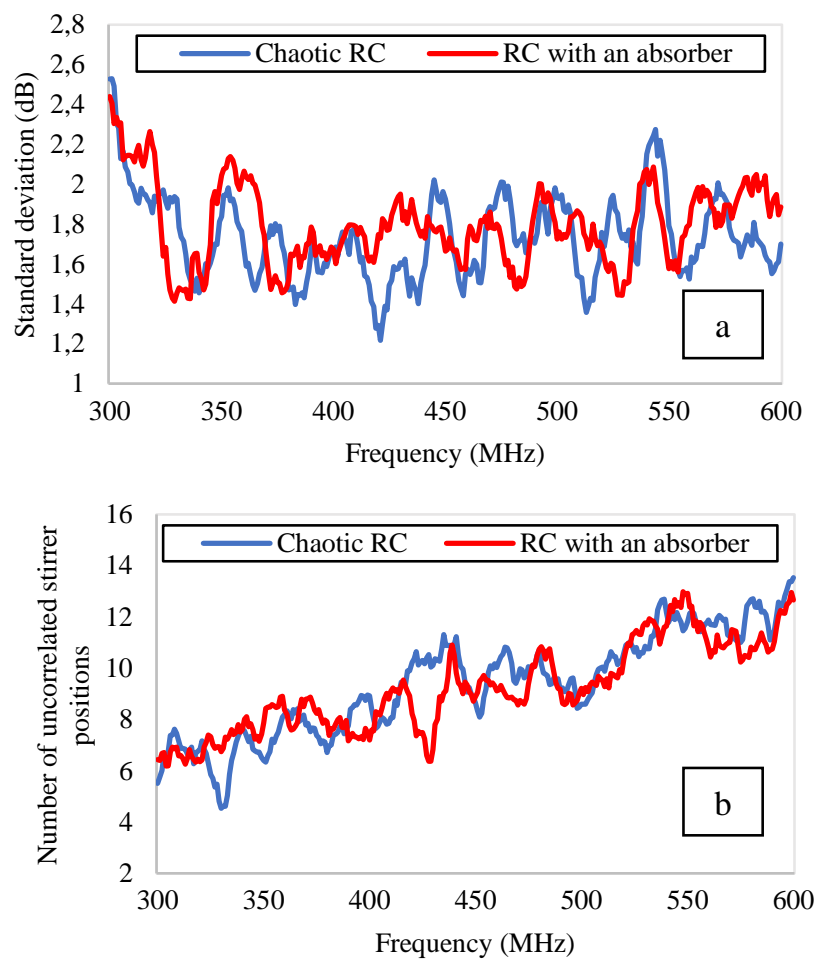

Fig.8. ESYCOM RC. (a) Standard deviation. (b) Number of uncorrelated stirrer positions. Results averaged over a $10 \mathrm{MHz}$ sliding window.
We define the distributions of the normalized intensities of the electric field as:

$$
P_{I}(I)=\int_{0}^{1} P_{\rho}(\rho) P(I ; \rho) d \rho
$$

with $\rho$ the phase rigidity and $I=\frac{\left|E_{a}\right|^{2}}{\left\langle\left|E_{a}\right|^{2}\right\rangle_{r}}$ the field component intensity both extracted from experimental data.

We draw the experimental and analytical cumulative density functions, noted $F(I)$, of the distributions of the normalized intensity of the electric field, in the cases of the $\mathrm{RC}$ containing an absorber, then in the chaotic one at the ESYCOM laboratory between 390 and $410 \mathrm{MHz}$ (Fig. 9 a) and between 980 and $1000 \mathrm{MHz}$ (Fig. 9 b).

We clearly observe an effect of the cavity geometry between 390 and $410 \mathrm{MHz}$ with a better agreement between experimental and theoretical distributions of the field intensity in the chaotic cavity. Indeed, the maximum difference between the analytical curve and the experimental one is about 0.04 in the $\mathrm{RC}$ with an absorber against 0.02 in the chaotic RC. Besides, in the frequency band $980-1000$ $\mathrm{MHz}$ the experimental distributions are close to theoretical ones in both cavity configurations ( 0.02 in both cavities) as at high frequencies the empty $\mathrm{RC}$ works properly.

We can conclude from this section that the addition of caps does not affect the normative criteria (number of uncorrelated stirrer positions and maximum E-field uniformity). However, an effect is observed on the intensity distributions and on the $C V$ at low frequency range $(300-$ $400 \mathrm{MHz}$ ), with a decrease of this ratio in the chaotic configuration.

\section{CONCLUSION}

In this communication, we have investigated the impact of geometrical modifications, inspired from the chaotic
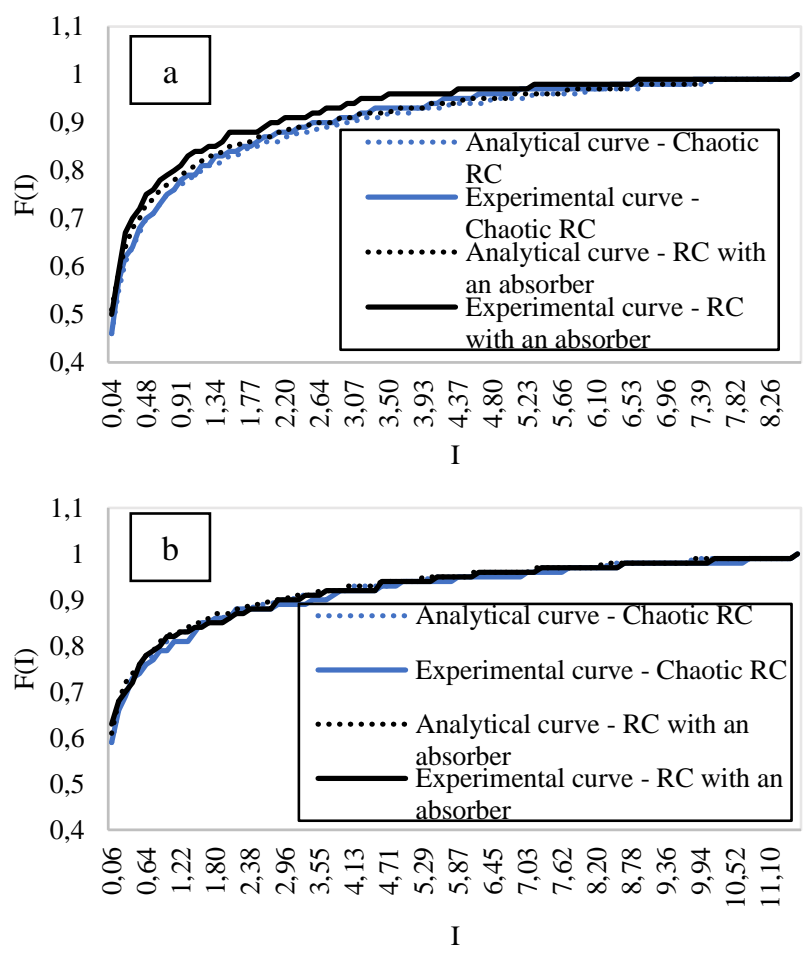

Fig.9. Cumulative density functions of the distributions of the normalized intensities of the electric field in the RC containing an absorber and the chaotic one at ESYCOM between $390-410 \mathrm{MHz}$ (a) and $980-1000 \mathrm{MHz}$ (b). 
cavity concept, on the field statistics within an RC. We showed that adding three metallic spherical caps on the RC walls enhances the field uniformity ( $C V$ closer to its ideal value). However, it also decreases the quality factor due to the loading effect. Thus, we compare the RC in its chaotic configuration (with the three caps) with the RC including an absorber so that its quality factor is similar compared to the one of the chaotic RC. We showed that the chaotic configuration presents a better field uniformity in the 300 $400 \mathrm{MHz}$ frequency range, according to the coefficient of variation. Beyond $400 \mathrm{MHz}$, the two cavities present the same behavior. The Pnini-Shapiro distribution confirms these results. It has to be noticed that the normative field standard deviation is much less sensitive than the $C V$.

We can conclude from the presented results that the addition of caps does not affect the normative criteria number of independent realizations and statistical uniformity of the field). However, an effect of the cavity geometry is observed on other criteria as the $C V$. Indeed, a geometry impact is seen when drawing the cumulative density functions of the distributions of the normalized intensity of the electric field.

This study also allows us to confirm that high absorption in an RC leads to an improvement of the modal overlap, $\mathrm{CV}$ and standard deviation but also results in a decrease in the number of uncorrelated stirrer positions. The $C V$ parameter is more sensitive than the other ones since based on an estimation of the first moments of a distribution with a large sample. A trade-off may be searched as regards the quantity of absorbing material to be added in an already chaotic cavity to reach a good field uniformity while reducing the $C V$ and keeping as much as independent stirrer positions as possible.

The results presented in this article correspond to measurements in different RCs leading to coherent conclusions.

\section{REFERENCES}

[1] J-F. Rosnarho, S. Le Berre, Reverberation Chambers Handbook, SIEPEL, version $4-2018$.

[2] P. Besnier, B. Démoulin, Electromagnetic Reverberation Chambers, ISTE/Wiley, Paris, 2011.

[3] IEC. "Electromagnetic Compatibility (EMC) Part 4-21: Testing and measurement techniques - Reverberation chamber test methods", NF EN 61000-4-21, October 2011.

[4] RTCA. "Environmental Conditions and Test Procedures for Airborne Equipment", RTCA DO-160G, December 2010.

[5] J.-B. Gros, U. Kuhl, O. Legrand, F. Mortessagne, "Lossy chaotic electromagnetic reverberation chambers: Universal statistical behavior of the vectorial field", Phys. Rev. E 93, 032108, (2016) DOI: 10.1103/PhysRevE.93.0332108.

[6] R. Pnini and B. Shapiro, "Intensity fluctuations in closed and open systems”, Phys. Rev. E 54, R1032, August 1996 https://journals.aps.org/pre/abstract/10.1103/PhysRevE.54.R1032.

[7] P. Besnier, C. Lemoine, J. Sol, "Various estimations of composite Qfactor with antennas in a reverberation chamber", Proc. IEEE International Symposium on Electromagnetic Compatibility (EMC), 2015, pp. 1123-1227.

[8] U. Kuhl, O. Legrand, F. Mortessagne, K. Oubaha, M. Richter, "Statistics of Reflection and Transmission in the Strong Overlap Regime of Fully Chaotic Reverberation Chambers", Invited Paper for session special sessions EMC at EUMCWeek 2017 in Nürnberg.

[9] C. Lemoine, P. Besnier, M. Drissi, "Estimating the effective sample size to select independent measurements in reverberation chamber", IEEE transactions on electromagnetic compatibility, vol. $50, n^{\circ} 2$, May 2008, pp 227-236. 